\title{
Pratique en santé publique au sein du système de soins de santé fournis aux militaires du Canada
}

\author{
Colonel Pierre Morissette ${ }^{1 *}$
}

Citation proposée : Morissette PJ. Pratique en santé publique au sein du système de soins de santé fournis aux militaires du Canada. Relevé des maladies transmissibles au Canada 2020;46(9):285-6.

https://doi.org/10.14745/ccdr.v46i09a01f

En tant que Canadiens, nous avons tant de raisons d'être reconnaissants, même si nous ne sommes peut-être pas toujours conscients de tous les avantages que nous tirons du simple fait de vivre dans un pays comme le Canada. La paix, la stabilité et la sécurité relatives qui sont inhérentes à la vie dans une nation arctique bordée sur trois côtés par les océans et sur le quatrième par une superpuissance mondiale amie se traduisent par l'exigence d'une armée modeste, au mieux, pour protéger le Canada et ses intérêts contre les agressions étrangères. Pourtant, même une armée modeste a besoin d'un système
Cette oeuvre est mise à la disposition selon les termes de la licence internationale Creative Commons Attribution 4.0

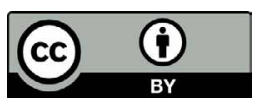

Affiliation

1 Direction de la protection de la santé de la Force, Services de santé des Forces canadiennes, Ottawa, ON (au moment de la rédaction de l'article)

${ }^{\star}$ Correspondance :

pierre.morissette@forces.gc.ca de santé capable de maintenir son personnel apte au service, avec la capacité de résoudre les problèmes de santé publique qui pourraient les menacer, que ce soit au pays ou à l'étranger. Ce qui suit est une brève introduction au système public de soins de santé fournis aux militaires du Canada, qui met l'accent sur la pratique de santé préventive qui se poursuit souvent en coulisses afin de maintenir l'état de préparation opérationnelle des militaires du Canada en optimisant la santé individuelle du personnel en uniforme.

Les Forces armées canadiennes (FAC) sont les services armés unifiés du Canada, qui comprennent les éléments maritimes, terrestres et aériens plus communément appelés la Marine royale du Canada, l'Armée canadienne et l'Aviation royale du Canada (1). Le personnel des FAC compte actuellement environ 101500 membres, dont 71500 membres à temps plein dans la Force régulière et 30000 membres à temps partiel dans la Force de réserve (1). Le personnel des FAC est appuyé par 25000 employés supplémentaires de la fonction publique civile du ministère de la Défense nationale (1).

La Loi constitutionnelle de 1867 établit l'autorité exclusive sur les questions qui portent sur « la milice, le service militaire et le service naval, et la défense » du Canada auprès du gouvernement fédéral (2). Ce pouvoir s'étend aux services de soins de santé pour le personnel des FAC qui sont expressément exclus de la définition de personnes assurées dans la Loi canadienne sur la santé (3). Le Groupe des Services de santé des Forces canadiennes (Gp Svc S FC), dirigé par le commandant du Gp Svc S FC et médecin général des Forces armées canadiennes, a pour objectif de combler cette lacune dans la Loi canadienne sur la santé en fournissant des services de santé au personnel des FAC partout au Canada ainsi qu'à ceux qui sont affectés ou déployés dans le cadre d'opérations militaires à l'étranger. De façon générale, le Gp Svc S FC offre des soins de santé complets au personnel des FAC comparables à ceux offerts à tous les autres Canadiens par leurs régimes de soins de santé provinciaux (4).

Le Gp Svc S FC est un système de soins de santé pancanadien ayant d'importantes responsabilités nationales et internationales, employant environ 6100 membres du personnel des services de santé et ayant un budget annuel de près de $471 \mathrm{M} \$$, excluant les salaires du personnel des FAC. Il collabore avec des organisations non gouvernementales et d'autres administrations de santé au niveau provincial et territorial et d'autres ministères fédéraux ayant des intérêts liés à la santé comme Santé Canada et l'Agence de la santé publique du Canada ainsi qu'avec des systèmes de santé qui leur sont propres, y compris Services aux Autochtones Canada et le Service correctionnel du Canada. Enfin, il collabore avec ses alliés militaires dans le cadre du Commandement de la défense aérospatiale de l'Amérique du Nord (NORAD), de l'Organisation du Traité de l'Atlantique Nord (OTAN) et des Nations Unies.

En ce qui concerne la médecine opérationnelle, le Gp Svc S FC doit être en mesure de fournir la « norme canadienne » en matière de soins de santé - $y$ compris la santé publique - aux membres des FAC dans l'ensemble des opérations militaires, de I'aide humanitaire aux secours en cas de catastrophe, en passant par le maintien de la paix et le combat. Dans un contexte militaire, la santé publique est souvent appelée «Protection de la santé des Forces », ce que définit l'OTAN comme suit: [traduction] « tous les efforts médicaux visant à promouvoir ou à 
conserver le bien-être physique et mental, à réduire ou à éliminer I'incidence et les répercussions des maladies, des blessures et des décès et à améliorer l'état de préparation opérationnelle et l'efficacité au combat des Forces » (5).

La composante de la santé publique du Gp Svc S FC consiste en des équipes multidisciplinaires de médecine préventive aux niveaux tactique (local) et opérationnel (régional) appuyées par des experts en la matière au sein d'un quartier général de niveau stratégique situé à Ottawa, connu sous le nom de la Direction - Protection de la santé de la Force (DPSF). Le DPSF est composé d'environ 60 personnes au total, y compris des experts militaires et civils et du personnel de soutien. Son rôle est de promouvoir la santé des membres des FAC ainsi que de prévenir les maladies et les blessures chroniques, les maladies infectieuses, les maladies professionnelles et environnementales, et de se préparer et d'intervenir en cas d'urgence en santé publique qui touche les FAC. Ces fonctions constituent la base de plusieurs programmes nationaux au sein du DPSF, y compris une capacité épidémiologique, un programme de promotion de la santé (aussi appelé Énergiser les Forces), un programme de santé au travail et de l'environnement, des équipes déployables de gestion des risques pour la santé, une capacité de service du renseignement médical et, bien sûr, un programme de contrôle des maladies transmissibles, qui sert de point de mire à ce thème du Relevé des maladies transmissibles au Canada.

Le DPSF entretient des partenariats avec des organismes de santé publique au sein du système de soins de santé civil canadien traditionnel, y compris le Conseil des médecins hygiénistes en chef (faisant partie du Réseau pancanadien de santé publique) ainsi qu'au sein de la communauté mondiale dans le cadre du Groupe de travail sur la protection de la santé des Forces de l'OTAN. Avec son programme de lutte contre les maladies transmissibles, la DPSF collabore avec les autorités locales de santé publique et apporte son expertise à plusieurs organismes de consultation hautement respectés, dont le Comité consultatif national de l'immunisation au Canada, le Comité consultatif de la médecine tropicale et de la médecine des voyages et le Groupe d'experts en renseignement médical de I'OTAN.

Un système de santé publique robuste est tout aussi essentiel pour les soldats, les marins et les aviateurs du Canada que pour tous les autres Canadiens. On sait que l'inaction des chefs militaires lors de la mise en œuvre des mesures de protection de la santé de leurs troupes peut nuire à l'état de préparation opérationnelle (6). L'histoire nous a montré à maintes reprises que la prévention des blessures et des maladies subies par les soldats en dehors du feu des combats est essentielle à la préservation de la puissance de combat militaire. Le personnel médical militaire canadien qui a participé à la Grande Guerre a noté que : [traduction] « La guerre actuelle a prouvé de façon très concluante que les fonctions de prévention et d'hygiène du service médical militaire sont plus importantes que les fonctions purement médicales » (7).

II s'ensuit que, pour s'assurer que le Canada demeure fort au pays, en sécurité en Amérique du Nord et engagé dans le monde, il est important que son armée relativement petite maintienne une capacité efficace en santé publique (8). Veuillez continuer à lire et prenez plaisir à en apprendre davantage sur la façon dont la santé publique est actuellement pratiquée dans le système de soins de santé fournis aux militaires du Canada et à contribuer aux réussites des FAC au pays et à l'échelle mondiale.

\section{Références}

1. Gouvernement du Canada. Loi sur la défense nationale (R.S.C. 1985, c. N-5) (modifié 2019-10-01; accédé 2019-1209). https://laws-lois.justice.gc.ca/fra/lois/N-5/TexteComplet. html

2. Gouvernement du Canada. Loi constitutionnelle de 1867 (modifié 2020-04-09; accédé 2019-12-09).

https://laws-lois.justice.gc.ca/fra/lois/N-5/TexteComplet.html

3. Gouvernement du Canada. Loi canadienne sur la santé (L.R.C. (1985), ch. C-6) (modifié 2017-12-12; accédé 201912-09). https://laws-lois.justice.gc.ca/eng/acts/C-6/FullText. html

4. Gouvernement du Canada. À propos de la Gamme de soins de santé (modifié 2019-01-15; accédé 2019-12-09). https:// www.canada.ca/fr/ministere-defense-nationale/services/ avantages-militaires/solde-pension-indemnites/prestations/ medicales-dentaires/generalites-administration.html

5. North Atlantic Treaty Organization. NATO Standard AJMedP-4, Allied Joint Medical Force Health Protection Doctrine, Ed. A v. 1, July 2018, p 1-1. https://www.coemed. org/files/stanags/02_AJMEDP/AJMedP-4_EDA_V1_E_2561. $\mathrm{pdf}$

6. Canadian Forces Joint Publication JP 4-10, Health Services Doctrine, Final Draft, March 2020, p 5-1. https://www.jcs.mil/ Portals/36/Documents/Doctrine/pubs/jp4_10.pdf

7. Adami JG. War Story of the Canadian Army Medical Corps. Vol 1. The First Contingent (to the Autumn of 1915). Published for the Canadian War Records Office. August 16, 1918 (accédé 2019-12-19). https://digital.library.upenn.edu/ women/adami/camc/camc.htm

8. Gouvernement du Canada. Protection, Sécurité, Engagement : La politique de défense du Canada. DN : 2017 (accédé 2020-01-21). https://www.canada. $\mathrm{ca} / \mathrm{fr} / \mathrm{ministere-defense-nationale/organisation/}$ rapports-publications/politique-defense-canada.html 